\title{
Cooling atoms in an optical trap by selective parametric excitation
}

\author{
N. Poli, R. J. Brecha, ${ }^{*}$ G. Roati, ${ }^{\dagger}$ and G. Modugno \\ INFM, LENS, and Dipartimento di Fisica, Università di Firenze, Largo E. Fermi 2, 50125 Firenze, Italy
}

(Received 3 July 2001; published 3 January 2002)

\begin{abstract}
We demonstrate the possibility of energy-selective removal of cold atoms from a tight optical trap by means of parametric excitation of the trap vibrational modes. Taking advantage of the anharmonicity of the trap potential, we either selectively remove the most energetic trapped atoms or excite those at the bottom of the trap by tuning the parametric modulation frequency. This process, which had been previously identified as a possible source of heating, also appears to be a robust way for forcing evaporative cooling in anharmonic traps.
\end{abstract}

DOI: 10.1103/PhysRevA.65.021401

PACS number(s): 32.80.Pj, 32.80.Lg

Parametric excitation of atomic motion due to laser intensity fluctuations has been recently indicated as one of the major sources of heating in far-off resonance optical traps (FORTs) [1,2], and as a limitation in achieving very long trapping times $[3,4]$. In this paper, we show that parametric excitation in a FORT can also lead to a cooling of the trapped sample, since the anharmonicity of the potential allows a selective removal of the most energetic atoms alone.

The excitation process has typically been modeled assuming a harmonic FORT potential $[1,2]$. For a modulation of the potential depth at twice the trap vibrational frequencies, an exponential heating of the trapped sample accompanied by trap losses has been predicted. The main features of the parametric heating process predicted by the harmonic theory have been experimentally observed [5-7]. Going beyond the basic trap model, it has been possible to further refine theoretical calculations through modeling of the anharmonic trapping potential used in actual experiments [8]. One of the consequences of the anharmonicity is a variation of the trap frequencies with vibrational energy, which implies that a modulation at a single frequency will be resonant with a few trap levels only.

We present here a detailed study of the temperature of a sample of cold atoms in a one-dimensional (1D) lattice FORT, in which the anharmonicity of the potential is exploited to perform energy-selective excitation and removal of the atoms, resulting in either heating or cooling of the remaining trapped sample. In particular, the cooling process appears to be a robust way to lower the atomic temperature via forced evaporation. One of the interesting aspects of this process is that the trap depth need not be reduced to force the evaporation, in contrast to the technique investigated for the first time in Ref. [9], and recently used to achieve BoseEinstein condensation [10]; the result is a tighter confinement of the cooled sample.

The trap is created with a beam of single-mode radiation generated by a Ti:sapphire laser at $\lambda_{T}=787 \mathrm{~nm}$, red detuned by approximately $18 \mathrm{~nm}$ from the $D$ lines of potassium. The laser beam, after passing through an acousto-optic modulator

\footnotetext{
*Permanent address: Physics Department, University of Dayton, Dayton, $\mathrm{OH}$.

${ }^{\dagger}$ Present address: Dipartimento di Fisica, Università di Trento, I-38050 Povo (Tn), Italy.
}

(AOM), is focused to $w=90 \mu \mathrm{m}$ within a two-lens telescope, and then retroreflected to form a vertical standingwave. The focusing is chosen to yield a large Rayleigh length $z_{R}=3 \mathrm{~cm}$, which results in an almost uniform lattice depth along the vertical direction. Therefore, the optical potential close to the beam waist has the form

$$
U(r, z)=-U_{0} \cos ^{2}(k z) \exp \left(-\frac{2 r^{2}}{w^{2}}\right)
$$

where $k=2 \pi / \lambda_{T}$. About $10^{7}$ atoms are prepared in a magneto-optical trap (MOT) at a temperature of $60 \mu \mathrm{K}$, and typically $5 \%$ of them are transferred to the FORT thanks to a compression procedure described in [11]. After loading the atoms in the FORT, the MOT beams are extinguished by means of AOMs and mechanical shutters, and the atoms are left to evolve in the presence of the trap light only. The density and the radial temperature of the trapped atoms are determined using absorption imaging of the atomic cloud after $1 \mathrm{~ms}$ of expansion following release from the FORT. The typical vertical size of the cloud of $700 \mu \mathrm{m}$ indicates that approximately 1800 lattice sites are occupied by an average of 300 atoms each. The axial temperature of the atoms is measured with a time-of-flight (TOF) technique, by absorption of a sheet of light passing about $1.5 \mathrm{~cm}$ below the FORT. The temperature of the trapped atoms, after a $100-\mathrm{ms}$ equilibration phase following loading, stays constant during storage in the FORT, because the heating due to photon scattering is compensated by a slow-evaporative cooling [11], and the radial and axial temperatures are found to be equal within the uncertainty. The lifetime of the FORT is limited to about $1.2 \mathrm{~s}$ mainly by collisions with background gas.

The harmonic approximations for the radial and axial frequencies of the single lattice site are measured with a parametric excitation technique, as described below. Typical values for these quantities are $\omega_{r}=2 \pi \times 1.3 \mathrm{kHz}, \omega_{a}=2 \pi$ $\times 700 \mathrm{kHz}$, which for the typical temperature $T=80 \mu \mathrm{K}$ lead to a density of $10^{12} \mathrm{~cm}^{-3}$. From the measured value of $\omega_{a}$, it is possible to estimate the trap depth as $U_{0}$ $=M \lambda_{T}^{2} \omega_{a}^{2} / 8 \pi^{2}$, which corresponds to $U_{0}=700 \mu \mathrm{K}$.

We apply the parametric excitation via the control AOM as a small sinusoidal modulation on the power of the trapping laser, typically $200 \mathrm{~ms}$ after loading the FORT. To measure the spectrum of excitation, we have repeated at different 


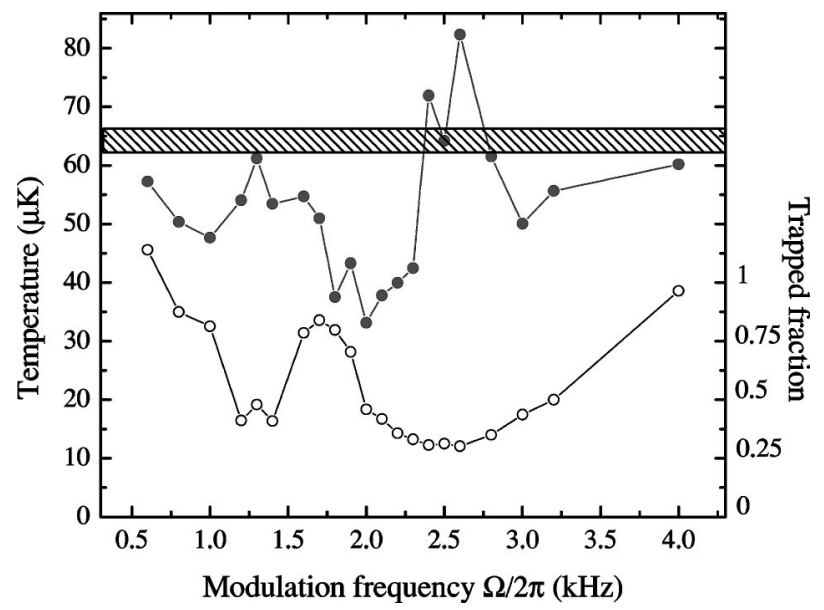

FIG. 1. Spectrum of the resonances in the trap losses (open circles) and in the radial temperature (solid circles) following a $T_{e x}=50 \mathrm{~ms}, \epsilon=30 \%$ excitation of the radial vibration. The shaded area indicates the initial temperature range, and represents the uncertainty in temperature.

modulation frequencies a procedure composed of an excitation for a period $T_{e x}$ at fixed modulation amplitude $\epsilon$ and frequency $\Omega$, followed by detection of the number of atoms and radial temperature. For both radial and axial excitation, as shown in Figs. 1 and 2, respectively, we see large resonances in the loss of atoms, accompanied by dispersive resonances in the temperature of the atoms left in the trap. The resonances in the trap losses have been observed previously in optical lattices $[5,8,11]$, and their presence is expected even if we approximate the FORT as a harmonic potential: when $\Omega$ is close to $2 \omega_{a} / n$ or $2 \omega_{r} / n$, with $n=1,2$, the atoms are excited upwards along the ladder of energy levels [1], and eventually lost from the trap. Note that for the axial excitation an additional resonance appears close to $4 \omega_{a}$, as expected for a sinusoidal (i.e., nonharmonic) potential with tight confinement [8].

On the other hand, the observed shape of the resonances in the temperature of the remaining trapped atoms are not expected for a harmonic trap $[1,2]$. Their origin must be re-

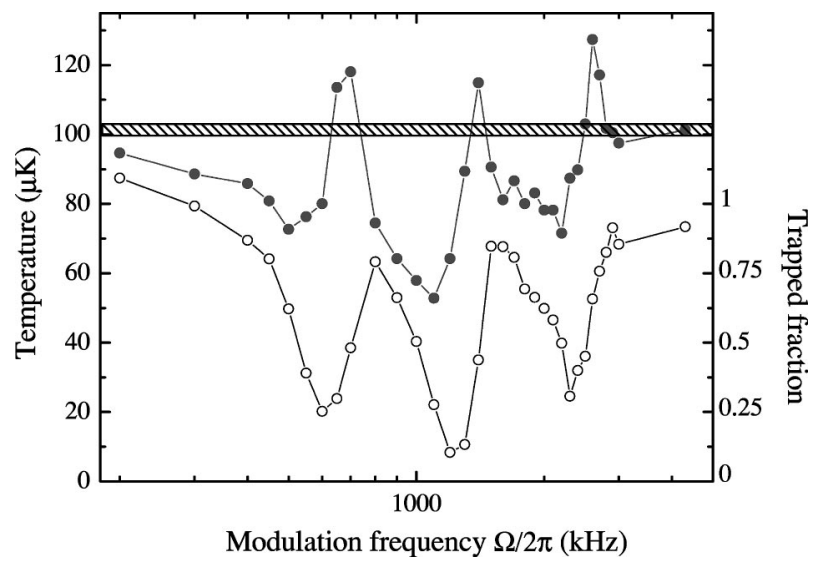

FIG. 2. Spectrum of the resonances in the trap losses (open circles) and in the radial temperature (solid circles) following a $T_{e x}=200 \mathrm{~ms}, \epsilon=16 \%$ excitation of the axial vibration.

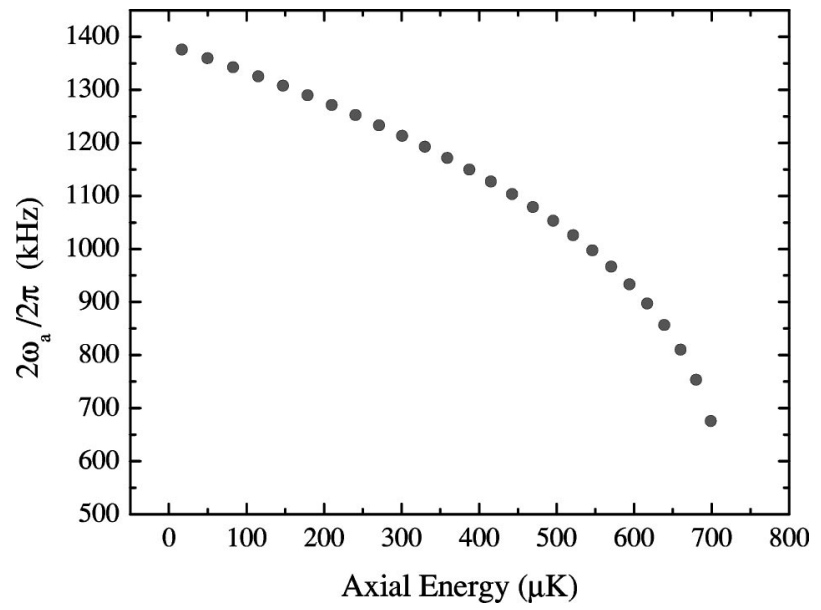

FIG. 3. Calculated spectrum of the axial excitation frequency for a $1 \mathrm{D}$ optical lattice with $U_{0}=700 \mu \mathrm{K}$.

lated to the anharmonicity of the potential, which implies that the vibrational frequency changes with atomic energy. In Fig. 3, we show the excitation frequency $2 \omega_{a}$ calculated for a perfectly 1D sinusoidal lattice: while the atoms at the bottom of the trap are expected to oscillate at the harmonic vibrational frequency, the most energetic ones have a much lower frequency, because of the decreased curvature of the potential. A similar behavior is also expected for the radial oscillation frequency because of the Gaussian shape of the radial potential. Therefore, when the modulation frequency is tuned to the red of $2 \omega_{r}, 2 \omega_{a}$ or their harmonics and subharmonics, it is mainly the most energetic atoms that are parametrically excited and ejected from the trap, resulting in a reduction of the mean energy of the remaining atoms. In contrast, modulation frequencies resonant with the nominal harmonic trap frequencies excite primarily atoms at the bottom of the trap, resulting in a net increase of the mean energy. We, therefore, identify the quantities $2 \omega_{r}, 2 \omega_{a}$ with the position of the main resonances in the temperature spectra of Figs. 1 and 2 leading to a heating of the trapped sample.

These observations are evidence of theoretical predictions that the peak in the resonance of atomic losses in the trap occurs at a slightly lower frequency than the actual harmonic frequencies [8]. This kind of information is very useful, since the frequencies measured from the trap-loss resonances are used to estimate quantities such as the number density or the phase-space density of atoms in an optical lattice $[5,11]$.

Note that all the temperature measurements reported in this paper have been performed on the radial degree of freedom only, since the TOF detection of the axial temperature was strongly perturbed by the atoms excited out of the trap as long as $100 \mathrm{~ms}$ before release. We have extracted the radial temperature, assuming thermal equilibrium from the width of the atomic distribution, which was always well fitted by a Gaussian.

In the following, we restrict our attention to the excitation of the axial vibrational mode, which is the most energetic and, therefore, requires a smaller perturbation of the trapping potential [12], making modeling easier. We also suspect that 


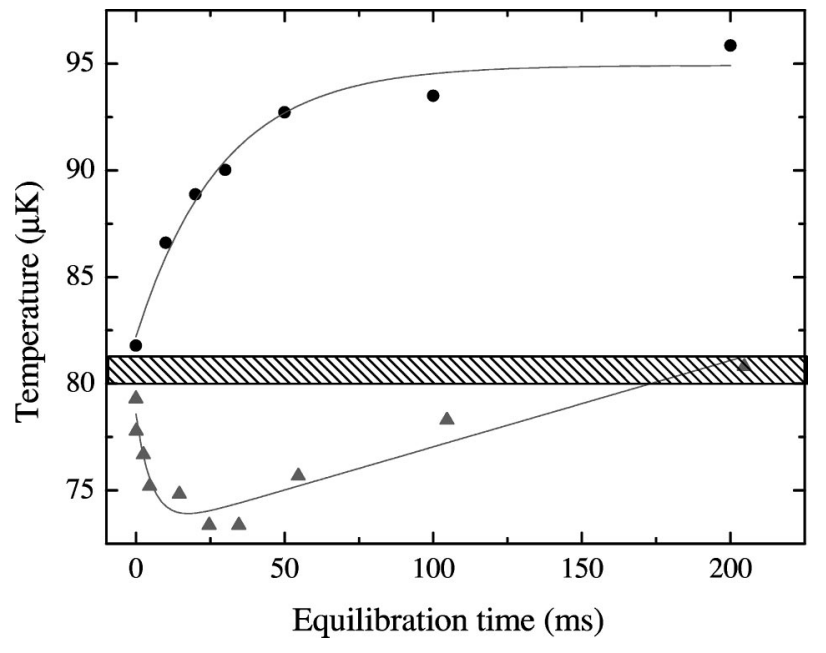

FIG. 4. Equilibration of the radial degree of freedom after an excitation at $1.8 \omega_{a}$ (triangles), with $T_{e x}=2 \mathrm{~ms}$ and $\epsilon=12 \%$, and at $2 \omega_{a}$ (circles), with $T_{e x}=10 \mathrm{~ms}$ and $\epsilon=12 \%$. The solid curves represent the best fit with an exponential equilibration, plus a constant heating rate for the lower set of data.

the large broadening of the radial resonances in Fig. 1 might come from a slight ellipticity in the cross section of the laser beam that makes $\omega_{r}$ vary in the radial plane, masking interesting characteristics of the resonances' shape.

To analyze in more detail the axial resonances, we need to refine the simple 1D model for the dispersion of the axial vibrational frequencies presented in Fig. 3. Indeed, when considering the actual potential of Eq. 1 one would expect that the axial oscillation frequency $\omega_{a}$ depends also on the total energy, and not only on the axial energy. Since the harmonic axial and radial oscillation periods differ by a factor of about 500, it is possible to define a local axial frequency that varies during the radial motion of the atoms [13]

$$
\omega_{a}(r)=2 \pi \sqrt{2 U_{0} \exp \left(-2 r^{2} / w^{2}\right) / M \lambda_{T}^{2}} .
$$

As a result, the discrete excitation frequencies shown in Fig. 3 for the 1D case become bands in 3D. The width of these levels is expected to be negligible for atoms at the bottom of the trap, which experience an harmonic potential, and to increase for increasing total energy, because of the longer time spent by the atoms in the anharmonic part of the potential. Thus, we expect that there is a broad range of frequencies on the red of the resonances where the cooling is effective, compared with a narrow range of frequencies in resonance where heating is present. These features seem to be qualitatively confirmed by the temperature spectrum in Fig. 2.

In addition, the anharmonicity of the potential might be responsible for a transfer of energy from the radial to the axial vibrational mode, after a parametric excitation phase has perturbed the equilibrium distribution. Indeed, after we have removed some of the axially energetic atoms with $2 \mathrm{~ms}$ of modulation at $\Omega=1.8 \omega_{a}$, the radial degrees of freedom appear to equilibrate on the time scale of $5 \mathrm{~ms}$, as reported in Fig. 4, which is almost an order of magnitude shorter than what expected for a thermalization mediated by collisions [11]. Moreover, we have observed that the equilibration rate

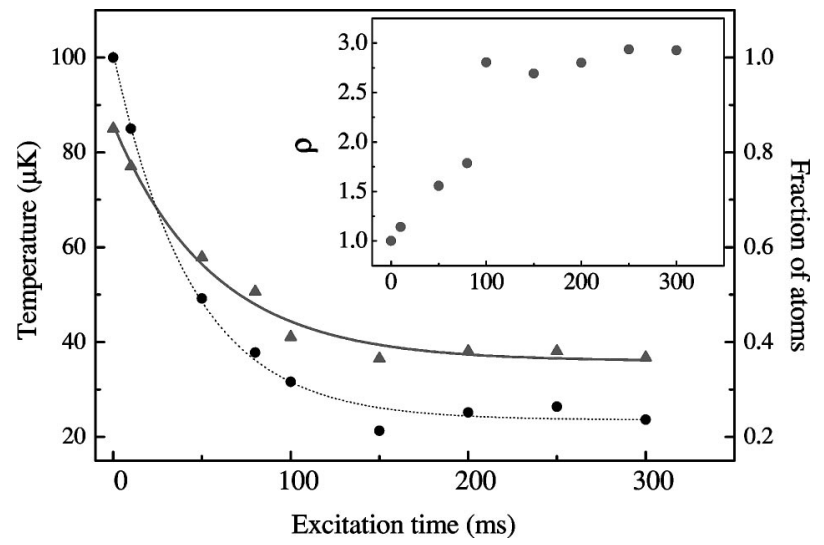

FIG. 5. Decrease of the radial temperature (triangles) and of the number of atoms (circles), during a parametric axial excitation with $\epsilon=12 \%$ and $\Omega=1.6 \omega_{a}$. The increase in relative phase-space density is shown in the inset

does not substantially vary when reducing the atomic density by a factor of 2 , indicating that cross-dimensional anharmonic mixing could actually favor a fast exchange of energy between the axial and radial modes of individual atoms in the trap [14]. On the contrary, as also reported in Fig. 4, when the atoms at the trap bottom are excited by tuning $\Omega$ to $2 \omega_{a}$, the two degrees of freedom equilibrate on a longer time scale of $20-50 \mathrm{~ms}$, which is more likely to be determined by the rate of interatomic collisions alone, and that indeed shows an almost linear dependence on the atomic density, as already observed in Refs. [11,15]. This result is expected, since the anharmonicity is not relevant for the atoms at the trap bottom. In conclusion, our heuristic model seems to predict a relatively fast removal of energy from all the three vibrational modes of the atoms, once a red-detuned axial modulation is applied continuosly.

We have investigated the time evolution of losses and temperature for modulation frequencies red detuned from $2 \omega_{a}$, to get an indication of the efficiency of the selective parametric excitation in cooling the atoms in the FORT. The evolution reported in Fig. 5 was obtained with $\epsilon=12 \%, \Omega$ $=1.6 \omega_{a}$, and $T_{e x}$ variable between 5 and $300 \mathrm{~ms}$; the atom number and temperature were detected after a $20-\mathrm{ms}$ thermalization period. The general features of the process discussed above are thus confirmed: at the beginning of the excitation phase the energetic atoms are very efficiently removed from the trap, and very likely the selection is performed on the total (3D) energy because of anharmonic mixing. We believe in any case that elastic collisions are responsible for a continuous replenishing of the high-lying states of the trap on a time scale much longer than $5 \mathrm{~ms}$, giving rise to the observed exponential decrease of both atom number and temperature. Thus, we can consider this process to be one of evaporative cooling, forced by the parametric excitation. As shown in Fig. 5, after $150 \mathrm{~ms}$ the atom number and temperature reach a steady-state value, which is conserved even after a further 150-ms period of excitation. The features just described are very interesting from the perspective of using this technique to cool the atoms in the trap, since an excitation at the proper frequency provides a fast 
removal of the energetic atoms without heating the coldest ones. After switching off the parametric modulation at $t$ $=300 \mathrm{~ms}$, we have measured a temperature increase at a rate of $34(12) \mu \mathrm{Ks}^{-1}$, compatible with that expected for photon scattering.

From the two sets of data in Fig. 5, we extract the evolution of the relative phase-space density $\rho$ reported the inset, assuming thermal equilibrium between radial and axial motions. Our experimental value for the parameter that describes the overall efficiency of an evaporative cooling process [16] is

$$
\gamma_{t o t}=\frac{N_{f} \rho_{f}}{N_{i} \rho_{i}}=0.7(1) .
$$

This efficiency, which could be limited also by the comparatively large heating rate due to photon scattering, can be compared to that of a variety of evaporative techniques in optical and magnetic traps $[10,16]$. To our knowledge the process we have investigated here represents the only way of forcing evaporative cooling in a standard optical trap [17] without a reduction in the trap depth.

Our FORT is actually not optimized for evaporation; indeed we are obliged by the short lifetime of the trap to force the removal of atoms on a time scale not much larger than the elastic collisional time. Further investigations in a better vacuum environment, or at a larger atomic density, can provide better tests of the efficiency of this cooling technique.
Indeed, with a longer time for evaporation one could try to use a modulation frequency on the red of all the axial resonances $\left(\Omega<\omega_{a}\right)$, to excite only the very energetic atoms and thus increase the cooling efficiency.

In conclusion, we have reported the observation of an energy-selective removal of atoms from a FORT, as a consequence of parametric excitation of anharmonic vibrational modes. In addition, we have shown that the energy selectivity can be exploited to cool the trapped sample. Since this effect arises due to the anharmonicity of the trapping potential, it should be observable in most actual traps for cold particles. The removal mechanism should work independently of the internal structure of the trapped particles (atoms, molecules or ions), as it is based on the excitation of their external degrees of freedom. The observations reported here should provide the impetus for further investigations and theoretical modeling necessary for characterizing the ultimate limits and efficiency of this cooling technique; however, it already appears interesting for a fast cooling of an atomic sample in a tight FORT.

We thank M. Inguscio for useful suggestions and for a careful reading of the manuscript. We also acknowledge useful discussions with R. Jáuregui, and we thank Scuola Normale Superiore, Pisa for the loan of the trapping laser. This work was supported by the ECC under Contract No. HPRICT1999-00111, and by MURST under the PRIN 2000 Program. R.B. was partially supported by ASI.
[1] T.A. Savard, K.M. O'Hara, and J.E. Thomas, Phys. Rev. A 56, R1095 (1997); M.E. Gehm et al., ibid. 58, 3914 (1998).

[2] C.W. Gardiner et al., Phys. Rev. A 61, 045801 (2000).

[3] K.M. O'Hara et al., Phys. Rev. Lett. 82, 4204 (1999).

[4] H. Engler et al., Phys. Rev. A 62, 031402 (2000).

[5] S. Friebel et al., Phys. Rev. A 57, R20 (1998).

[6] V. Vuletic et al., Phys. Rev. Lett. 81, 5768 (1998).

[7] J. Ye, D.W. Vernooy, and H.J. Kimble, Phys. Rev. Lett. 83, 4987 (1999).

[8] R. Jáuregui et al., Phys. Rev. A 64, 033403 (2001).

[9] C.S. Adams et al., Phys. Rev. Lett. 74, 3577 (1995).

[10] M.D. Barrett, J.A. Sauer, and M.S. Chapman, Phys. Rev. Lett. 87, 010404 (2001).
[11] G. Roati et al., Phys. Rev. A 63, 052709 (2001).

[12] A general result of the theory presented in Ref. [1] is that the excitation rate is proportional to $\epsilon^{2} \omega^{2}$.

[13] T. Elsässer, Diploma thesis, University of Heidelberg, 2000.

[14] We have labeled the ordinate axis of Fig. 4 as "temperature," since we are not able to distinguish an equilibration mediated by mixing from a thermalization mediated by collisions.

[15] V. Vuletic et al., Phys. Rev. Lett. 82, 1406 (1999).

[16] W. Ketterle and N.J. van Druten, Adv. At., Mol., Opt. Phys. 37, 181 (1996).

[17] An exception is represented by the optical trap investigated in K.L. Corwin et al., Phys. Rev. Lett. 83, 1311 (1999). 